\title{
Behavioural states in normal mature human fetuses
}

\author{
M Pillai, D James
}

\begin{abstract}
Behaviour was studied by real time ultrasound in a group of 80 low risk fetuses between 36 and 42 weeks' gestation. There was close linkage of fetal eye movements, somatic movements and heart rate pattern reflecting three different states. Quiescence (state 1F) was characterised by no eye movements, no somatic movements except for the occasional startle, and a fetal heart rate pattern with little baseline variability. There were two active states: state $2 \mathrm{~F}$ characterised by continuous eye movements, frequent bursts of somatic movements, and wide baseline variability with accelerations with movement, and state $4 F$ characterised by continuous eye movements with almost continuous somatic movements and a sustained tachycardia. These fulfilled criteria for three of four behavioural states previously described in human fetuses. Cycling of quiescent and active states occurred in $77(96 \%)$ of the fetuses within 100 minutes of starting the recording. $2 \mathrm{~F}$ was the commonest behavioural state, being seen $58 \%$ of the time. 1F was seen $30 \%$ of the time, and $4 F 9 \%$ of the time. During the remaining $3 \%$ of observation time the behavioural state was indeterminate.
\end{abstract}

For several years we have known that healthy newborn infants normally manifest least five different behavioural states: quiet sleep (S1), active/rapid eye movement (REM) sleep (S2), quiet awake (S3), and active awake without crying (S4), and with crying (S5). These are characterised by recurring conditions of certain variables in stable association with one another. These variables include eye movements, heart rate pattern, breathing pattern, and electroencephalographic pattern and activity. ${ }^{1}$ For practical reasons the criteria used for the states in the newborn are unsuitable for the fetus. Unlike the infant the fetus does not make breathing movements all the time, and opening and closing of the eyes cannot always be observed easily with ultrasound, nor can an electroencephalogram be recorded until after rupture of the membranes. In 1981, however, Bots et al validated the detection of fetal eye movements with ultrasound and it was this that made the study of fetal behavioural states possible. ${ }^{2}$

Using real time ultrasound recordings of fetal motility with simultaneous continuous recording of the fetal heart rate, Nijhuis $e t$ al analysed the temporal association of different types of somatic movements, eye movements, and heart rate patterns of fetuses of multiparous women that were between 32 and 40 weeks' gestation. ${ }^{3}{ }^{4}$ After 36 weeks they found distinct, recurring combinations of these variables that they considered analagous to the first four of the five behavioural states described in infants (S1 to S4), and they therefore designated them states $1 \mathrm{~F}$ to $4 \mathrm{~F}$. Arduini et al have studied the distribution of fetal breathing, eye movements, and micturition during active and quiet cycles throughout the last trimester, ${ }^{5}$ but there have been no detailed studies of the variations and consistencies in behaviour of healthy or compromised fetuses.

The aim of this study was to document variations in behaviour in a group of healthy mature fetuses.

\section{Patients and methods}

Forty primigravidas and 42 multigravidas with uncomplicated singleton pregnancies of at least 36 completed weeks' gestation were studied. The observations were conducted in a quiet room between the hours of 10 am and $6 \mathrm{pm}$. In each case a comfortable semirecumbent position with some lateral tilt was used, and for this purpose we found a Parker Knoll reclining chair was most suitable. The aim was to undertake recordings for at least 100 minutes, because from pilot studies this period of time would allow for at least one cycle of quiet/active behaviour in over $95 \%$ of observations (unpublished data). This was not possible in 12 cases (recording time 74-90 minutes) because the mother asked us to stop earlier. All 12 cases had cyclical activity, and there was no evidence that the slightly suboptimal recording times resulted in differences in behavioural findings.

Initial measurements of biparietal diameter, head circumference, and abdominal circumference were noted together with an assessment of liquor volume and fetal normality to confirm that these were low risk pregnancies.

Fetal eye, head, and upper limb movements were detected by one real time ultrasound transducer (Siemens Sonoline SLl, 3.5 MHz transducer or Toshiba SAL 20A, $2 \cdot 4 \mathrm{MHz}$ transducer) and were stored on video tape, and fetal trunk movements were visualised on a transverse view of the fetal abdomen detected by a second transducer (Toshiba SAL 20A, 2.4 $\mathrm{MHz}$ transducer) and recorded verbally on the sound channel of the video recorder. The fetal heart rate was simultaneously recorded with a cardiotocograph (Hewlett Packard 8040A) at a paper speed of $1 \mathrm{~cm} /$ minute. All movements and the heart rate were additionally recorded on a polygraph at a paper speed of $3 \mathrm{~cm} /$ minute using multiple hand held push buttons, and the 


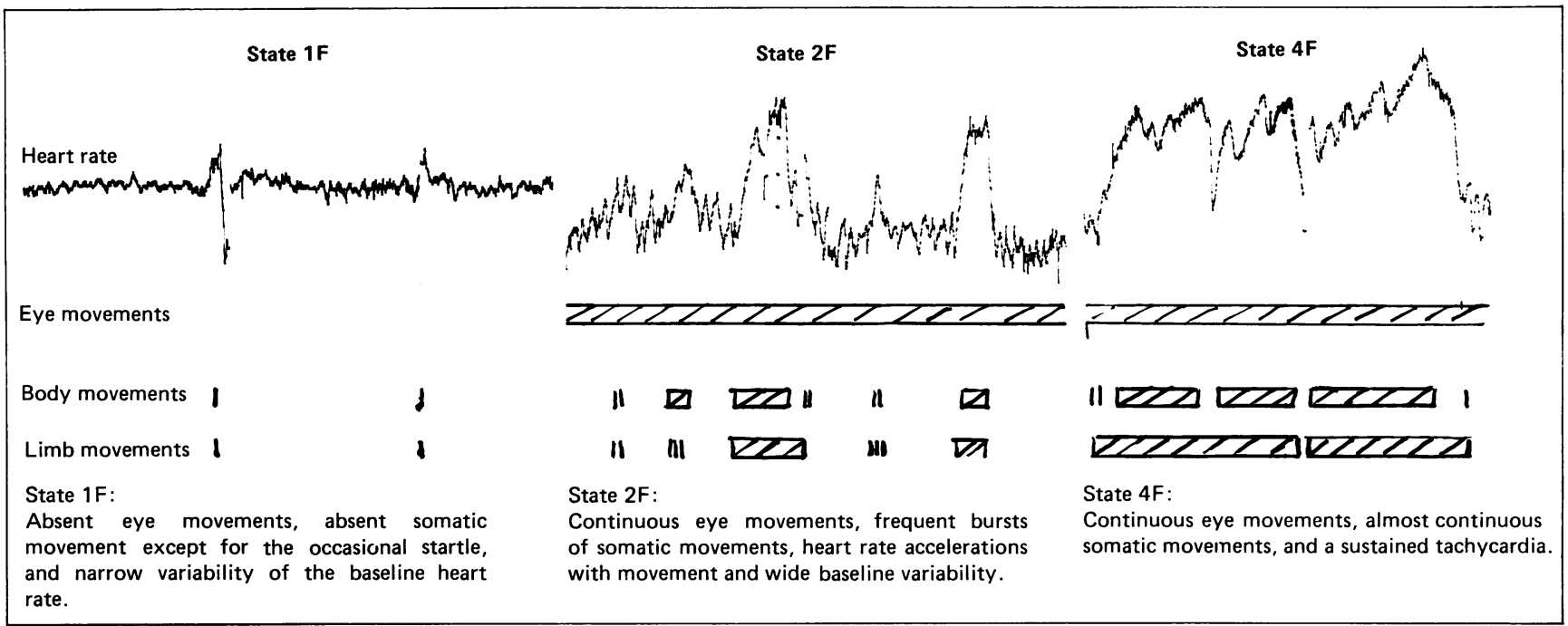

Fetal actogram at 39 weeks' gestation.

accuracy of this technique was checked on subsequent playback of the video tape. With this configuration of equipment little interference was obtained using all three transducers simultaneously.

The recordings were used to ascertain the association of the fetal heart rate pattern, eye movements, and somatic movements. The baseline variability of the heart rate pattern was calculated as the mean amplitude of oscillations in the baseline heart rate pattern.

Statistical comparisons between the states, gestational age, time of day, and characteristics of the different heart rate patterns were made by analysis of variance. Ethical approval was obtained from the hospital's research and ethics committee.

\section{Results}

Two recordings had to be excluded from analysis of behaviour patterns because visualisation of fetal eye movements was unsatisfactory for 70 to $80 \%$ of the recording. The analysis is therefore of the data from 80 fetuses. The total duration of observation sessions was $138 \cdot 2$ hours (mean 103.7 minutes, range 74 to 137 minutes/ recording).

BEHAVIOURAL PATTERNS

We identified three different behavioural patterns - two active patterns $(2 \mathrm{~F}$ and $4 \mathrm{~F})$, and one quiescent pattern (1F) (figure). Seventy seven of the 80 fetal recordings contained alternating cycles of quiescence and activity. In the remaining three recordings the fetuses sustained a high degree of activity throughout.

\section{Quiescence (state $1 F$ )}

A total of 121 episodes of quiescence were recorded (table 1). Three fetuses did not have an episode of quiescence during the recording interval. Seventeen recordings contained an episode of quiescence lasting 30 minutes or more, and the maximum duration recorded was 38 minutes (once in each of two recordings). In 44 recordings more than one episode of quiescence occurred and these have been examined for variability between fetuses.

Characteristics of the accompanying heart rate pattern are shown in table 2 . The baseline heart rate and its variability were not always consistent throughout an episode of quiescence or between successive episodes, and the average values for each fetus have been estimated. Ninety three episodes of quiescence were interrupted by startles, and 28 episodes were completely quiescent (the longest episode of complete quiescence being 38 minutes). The mean frequency of startles was one every 8.5 minutes, but within individuals startles seemed to occur at random. Startles were nearly always accompanied by an acceleration in fetal heart rate. There were no eye movements during

Table 1 Number and duration of each behavioural state in 80 fetuses of 36 to 42 weeks' gestation

\begin{tabular}{|c|c|c|c|c|c|}
\hline & \multicolumn{4}{|c|}{ Behavioural state } & \multirow[b]{2}{*}{ Total } \\
\hline & $1 F$ & $2 F$ & $4 F$ & Indeterminate & \\
\hline $\begin{array}{l}\text { No of episodes } \\
\text { Mean (SD) duration of episode (mins) } \\
\text { Range }\end{array}$ & $\begin{array}{l}121 \\
20 \cdot 8(8 \cdot 6) \\
3-38\end{array}$ & $\begin{array}{l}150 \\
31 \cdot 6 \\
3-94\end{array}$ & $\begin{array}{l}21 \\
37 \cdot 8(30 \cdot 4) \\
4-137\end{array}$ & $\begin{array}{l}25 \\
9 \cdot 2(5 \cdot 6) \\
3-20\end{array}$ & $\begin{array}{l}317 \\
26 \cdot 1(18 \cdot 1) \\
3-137\end{array}$ \\
\hline Total (\%) time spent in each state (mins) & $2516(30 \cdot 2)$ & $4785(57 \cdot 5)$ & $794(9 \cdot 5)$ & $231(2 \cdot 8)$ & 8326 \\
\hline
\end{tabular}

State $1 \mathrm{~F}=$ no eye movements, no somatic movement except for the occasional startle, and narrow variability of the baseline heart rate. State $2 \mathrm{~F}=$ continuous eye movements, frequent bursts of somatic movements, heart rate accelerations with movement, and wide baseline variability. State $4 \mathrm{~F}=$ continuous eye movements, almost continuous somatic movements, and a sustained tachycardia. 
Table 2 Characteristics of heart rates in each behavioural state

\begin{tabular}{|c|c|c|c|c|}
\hline & \multicolumn{4}{|c|}{ Behavioural state } \\
\hline & $\overline{l F(n=77)}$ & $2 F(n=79)$ & $p$ Value* & $4 F(n=16)$ \\
\hline $\begin{array}{l}\text { Mean (SD) baseline heart rate (beats/min) } \\
\text { Range } \\
\text { Mean (SD) baseline variability (beats/min) } \\
\text { Range }\end{array}$ & $\begin{array}{l}133 \cdot 1(8 \cdot 3) \\
115-150 \\
5 \cdot 4(2 \cdot 0) \\
2-10\end{array}$ & $\begin{array}{c}133 \cdot 5(7 \cdot 9) \\
115-153 \\
11 \cdot 4(2 \cdot 9) \\
4-19\end{array}$ & $\begin{array}{c}0.38 \\
<0.001\end{array}$ & $\begin{array}{l}160 \cdot 1(3 \cdot 7) \\
155-172 \\
\text { Sustained tachycardia } \\
\text { not baseline rate } \\
\text { so variability not } \\
\text { analysed }\end{array}$ \\
\hline
\end{tabular}

State $1 \mathrm{~F}=$ no eye movements, no somatic movement except for the occasional startle, and narrow variability of the baseline heart rate. State $2 \mathrm{~F}=$ continuous eye movements, frequent bursts of somatic movements, heart rate accelerations with movement, and wide baseline variability. State $4 \mathrm{~F}=$ continuous eye movements, almost continuous somatic movements, and sustained tachycardia.

*Analysis of variance, $1 \mathrm{df}$.

episodes of quiescence except occasionally during startles. These intervals of quiescence fulfil the criteria for behavioural state $1 F$ described previously. ${ }^{34}$

\section{Activity (states $2 F$ and $4 F$ )}

A total of 171 active cycles were observed, and accounted for $67 \%$ of the total recording time (table 1). The most common behavioural pattern was characterised by frequent bursts of body and limb movements lasting for $20-40$ seconds, with intervals of 40-80 seconds between each burst, and occasionally frequent isolated movements. Bursts of movement were nearly always accompanied by an acceleration in fetal heart rate. Between bursts of movement the baseline heart rate was similar to that of the preceding or succeeding quiescent cycle, but within individuals the baseline variability was significantly wider than during quiescence $(\mathrm{p}<0.001)$. Between individual fetuses there was considerable variation in the baseline rates and variability of the different states, which precluded use of numerical definitions.

There were no significant differences among gestational ages within the range 36 to 42 weeks (table 3).

Three fetuses had baseline heart rates and variability that were similar in activity and quiescence, but in two the different states were still readily distinguishable by the more regular oscillations of the baseline value in quiescence compared with that during activity. In the remaining fetus such episodes could only be distinguished by consideration of eye and somatic movements as well. Sixteen fetuses had one or more intervals of sustained almost continuous activity, with the accompanying heart rate accelerations tending to become fused to a sustained tachycardia, so obscuring the baseline value. These episodes accounted for $9 \%$ of the total recording time. In one fetus this high degree of activity persisted for the entire duration of recording so it was extended to determine the maximum length of such intervals, but had to be abandoned after 137 minutes. These two types of active behavioural patterns fulfil the criteria for states $2 \mathrm{~F}$ and $4 \mathrm{~F}$ previously described. ${ }^{3} 4$

\section{Indeterminate behaviour}

During $3 \%$ of the study time fetal behaviour was indeterminate, the variables not conforming to any one of the recognised states for a least three consecutive minutes. About half of these episodes occurred at the time of transition between different states.

\section{Other behavioural patterns}

Previous authors have described a fourth behavioural state-' $3 F$ '-in which eye movements are present, there are no somatic movements, and the heart rate pattern has a wider baseline variability than in $1 \mathrm{~F}$ but a more regular oscillation frequency than in $2 \mathrm{~F}^{3}{ }^{3} \mathrm{We}$ were unable to confirm the existence of this state in the human fetus. Indeed in $\mathbf{1 3 8}$ hours of recording there was only one interval in which eye movements were not associated with somatic movements for three minutes or more. This epoch lasted for four minutes. It was observed during a sustained period of $2 \mathrm{~F}$, and the accompanying fetal heart rate pattern had a baseline oscillation frequency that was characteristic of-and indistinguishable from-that of the preceding and succeeding $2 \mathrm{~F}$ fetal heart rate pattern.

\section{State transitions}

It has been suggested that before a fetus can be

Table 3 Trends in incidence of behavioural states and heart rates by gestational age from 36 to 42 weeks

\begin{tabular}{|c|c|c|c|c|}
\hline & \multicolumn{4}{|c|}{ Gestational age (weeks) } \\
\hline & $36-37(n=15)$ & $37-38(n=24)$ & $38-39(n=19)$ & $>39(n=22)$ \\
\hline $\begin{array}{l}\text { Mean (SD) percentage in state } 1 \mathrm{~F} \\
\text { Mean (SD) percentage in state } 2 \mathrm{~F} \\
\text { Mean (SD) percentage in state 4F } \\
\text { Mean (SD) percentage in indeterminate state } \\
\text { Mean (SD) basal heart rate in state } 1 \mathrm{~F} \\
\text { Mean (SD) basal heart rate in state } 2 \mathrm{~F} \\
\text { Mean (SD) baseline variability in state } 1 \mathrm{~F} \\
\text { Mean (SD) baseline variability in state } 2 \mathrm{~F}\end{array}$ & $\begin{aligned} & 28 \cdot 6(9 \cdot 5) \\
& 60 \cdot 5(12 \cdot 3) \\
& 7 \cdot 0(15 \cdot 0) \\
& 2 \cdot 3(5 \cdot 8) \\
& 132 \cdot 4(9 \cdot 4) \\
& 131 \cdot 5(7 \cdot 0) \\
& 6 \cdot 7(2 \cdot 2) \\
& 11 \cdot 1(3 \cdot 1)\end{aligned}$ & $\begin{aligned} & 29 \cdot 7(15 \cdot 0) \\
& 55 \cdot 8(16 \cdot 7) \\
& 11 \cdot 4(20 \cdot 3) \\
& 3 \cdot 2(5 \cdot 3) \\
& 133 \cdot 4(8 \cdot 2) \\
& 13 \cdot 5(8 \cdot 7) \\
& 4 \cdot 7(2 \cdot 1) \\
& 10 \cdot 8(3 \cdot 0)\end{aligned}$ & $\begin{aligned} 31 \cdot 2(11 \cdot 7) \\
60.1(17 \cdot 7) \\
6 \cdot 2(16 \cdot 9) \\
4 \cdot 8(5 \cdot 5) \\
133.0(8 \cdot 4) \\
134 \cdot 5(7 \cdot 9) \\
5 \cdot 2(1 \cdot 5) \\
12 \cdot 6(2 \cdot 2)\end{aligned}$ & $\begin{array}{r}33 \cdot 3(13 \cdot 7) \\
52 \cdot 5(23 \cdot 8) \\
10 \cdot 2(26 \cdot 6) \\
1 \cdot 1(2 \cdot 7) \\
133 \cdot 5(7 \cdot 9) \\
134 \cdot 3(7 \cdot 8) \\
5 \cdot 3(2 \cdot 0) \\
11 \cdot 3(3 \cdot 0)\end{array}$ \\
\hline
\end{tabular}

State $1 \mathrm{~F}=$ no eye movements, no somatic movement except for the occasional startle, and narrow variability of the baseline heart rate. State $2 \mathrm{~F}=$ continuous eye movements, frequent bursts of somatic movements, heart rate accelerations with movement, and wide baseline variability. State $4 \mathrm{~F}=$ continuous eye movements, almost continuous somatic movements, and sustained tachycardia.

There are no significant differences among the groups. 
Table 4 Influence of recording time on behaviour

\begin{tabular}{lllll}
\hline & \multicolumn{2}{l}{ Behavioural state } & & \\
\cline { 3 - 5 } & $I F$ & $2 F$ & $4 F$ & Indeterminate \\
\hline $\begin{array}{l}\text { Mean (SD) percentage time spent in each state } \\
\text { by each fetus }\end{array}$ & & & & \\
$\begin{array}{l}\text { Morning }(\mathrm{n}=35) \\
\text { Afternoon }(\mathrm{n}=45)\end{array}$ & $29 \cdot 8(13 \cdot 0)$ & $57 \cdot 7(16 \cdot 9)$ & $9 \cdot 1(17 \cdot 7)$ & $2 \cdot 8(4 \cdot 5)$ \\
\hline
\end{tabular}

State $1 \mathrm{~F}=$ no eye movements, no somatic movement except for the occasional startle, and narrow variability of the baseline heart rate. State $2 \mathrm{~F}=$ continuous eye movements, frequent bursts of somatic movements, heart rate accelerations with movement, and wide baseline variability. State $4 \mathrm{~F}=$ continuous eye movements, almost continuous somatic movements, and sustained tachycardia. There are no significant differences among the groups.

considered to have behavioural states, the transitions from one state to the next should not take longer than three minutes. ${ }^{34}$ We felt that is was only valid to consider transitions into and out of $1 \mathrm{~F}$ (quiescence), as only these transitions require a change in the condition of all three variables. We observed 181 such transitions (11 transitions in which there was poor visibility of the fetal eye have not been included) and found that these transition times varied between 5 and 1320 seconds. We noted a trend towards shorter transition times with advancing gestation within the ages studied, but these differences were not significant $(\mathrm{p}=0.572)$.

Individual fetuses showed considerable variation in transition times between states ( 5 to 520 seconds). In view of this variation we considered that to implement a 'three minute transition time' rule for distinguishing fetuses with and without behavioural states was arbitrary, unnecessarily complicated, and confusing. Ignoring the 3 minute transition rule, we found a high percentage of linkage between the variables as behavioural states, and a low incidence of indeterminate behaviour (table 1).

\section{EYE MOVEMENT}

We found that fetal eye movements could be recognised easily with a little practice, but unsatisfactory visualisation of the fetal eye was the main limitation in the recording and analysis of fetal behavioural states, resulting in our being unable to study two of the 82 fetuses at full term. This problem usually occurred when the fetal head was in the direct occipitoanterior position, when it was quite impossible to visualise the eye even with the head unengaged. Conversely when the head was in the occipitotransverse or occipitoposterior positions, good visualisation could be obtained even when the head was deeply engaged.

TIME OF DAY

We did not attempt to examine diurnal variations in behavioural states, but over the range of times included, recordings beginning before and after noon fell into two groups of similar size, and these were compared (table 4). The only apparent trend was for more quiescence (1F) in morning than afternoon studies, but this was not significant.

NEONATAL OUTCOME

All birth weights were between the 10th and 90th percentile for sex and gestational age. All infants had Apgar scores at one minute above 5 , and Apgar scores at five minutes above 8. None required admission to the neonatal intensive care unit, and the only neonatal problem before discharge was moderate jaundice in two infants, which was treated with phototherapy.

\section{Discussion}

The potential importance of accurate documentation of behavioural patterns and their development in the human fetus lies in enhancement of our understanding of the aetiology and pathophysiology of neurodevelopmental handicap that develops before birth.

The main clinical implication of these studies is that they emphasise the need to redefine normal limits for the biophysical tests of fetal well being currently in use. For example, conventional biophysical profile scoring requires three fetal movements and two fetal heart rate accelerations in a 30 minute period. ${ }^{6}$ Twelve of the 80 recordings in this study contained episodes of $1 \mathrm{~F}$ lasting 30 minutes or longer without two or more startles, and these healthy fetuses would not have achieved a normal score had testing occurred during these intervals. In contrast, three movements should occur in minutes during an interval of $2 \mathrm{~F}$, and in seconds during an interval of $4 \mathrm{~F}$. The same limitations apply to the non-stress test.

Recently a number of papers about auditory stimulation of the fetus have been published, but only one group of authors have looked at results in relation to the fetal behavioural state; they found that the results were dependent on behaviour state. ${ }^{7}$ The indices of Doppler recording of fetal blood flow also seem to be dependent on behaviour state. ${ }^{8-10}$ The importance of recognising this dependence is well established in neurological assessment of the newborn and should not be ignored in any tests involving stimulation of the fetus. ${ }^{1-13}$

We have confirmed the existence of three of the four behavioural states previously described in a group of 80 healthy fetuses of 36 or more completed weeks' gestation. We were unable, however, to find a state $3 \mathrm{~F}$ (eye movements present, no body movements, and a fetal heart rate pattern with a wider oscillation frequency than in $1 \mathrm{~F}$ but with more regular oscillations than in $2 F$ ) in any of the recordings. To explain this we considered whether the use of a paper speed of $1 \mathrm{~cm} /$ minute (the standard recording speed used in most units in the United Kingdom) rather than $3 \mathrm{~cm} /$ minute (as used in The Netherlands) might account for our failure to differentiate this heart rate pattern. As patterns accompany- 
ing states $1 \mathrm{~F}, 2 \mathrm{~F}$, and $4 \mathrm{~F}$ could clearly be differentiated, however, and were exactly as described in recordings performed at $3 \mathrm{~cm} /$ minute, we felt that this pattern should have been distinguishable. Furthermore, we have carried out some recordings subsequently at a paper speed of $3 \mathrm{~cm} /$ minute and found that the differences between patterns accompanying the different states are if anything less apparent. We still cannot recognise this pattern.

The more likely explanation for our failure to find state $3 \mathrm{~F}$ is that it occurs infrequently, if at all, in the fetus. We note that in previous studies it was found in only a minority of fetuses, and for short intervals, typically of three to six minutes duration. ${ }^{3} 4$

The main practical problem we encountered was our inability to observe fetal eye movements when the fetal head was in the direct occipitoanterior position, and we could not find any means of overcoming this except by waiting for the fetus to change position.

Consideration of ultrasonically recorded measurements as either linked behavioural patterns ('states') or indeterminate behaviour, rather than 'states', 'coincidence' and 'nocoincidence' as described by previous authors, ${ }^{34}$ simplifies the analysis and allows interpretation of shorter recordings, which might be of greater clinical relevance. It is also likely to make interpretation of recordings at earlier gestational ages easier, when linkage between the variables is less consistent (unpublished observations).

Clearly these behavioural cycles-of what seem similar to quiet sleep and active states in the newborn-are well developed in the normal human fetus at full term, and may indicate integrity and maturity of neurological development quite unlike any other biophysical investigations. For the time being the clinical applicability of such recordings is limited, firstly because both the recordings and their analysis are extremely time consuming, and secondly availability of all the ultrasound equipment required is likely to be limited.
As yet, however, there is little information on the sequence of normal development of behaviour, the influence of antenatal complications, and the association with long term neurodevelopmental outcome. For these reasons such behavioural studies remain of central importance in the research of perinatal neurological development.

This study was funded by grant A/8/1645 from Action Research for the Crippled Child.

1 Prechtl HFR. The behavioural states of the newborn infant. Brain Res 1974;76:185-212.

2 Bots RSGM, Nijhuis JG, Martin CB Jr, Prechtl HFR. Human fetal eye movements: detection in utero by ultrasonography. Early Hum Dev 1981;5:87-94.

3 Nijhuis JG, Prechtl HFR, Martin CB Jr, Bots RSGM. Are there behavioural states in the human fetus? Early Hum Dev 1982;6:177-95.

4 Nijhuis JG, Martin CB Jr, Prechtl HFR. Behavioural states of the human fetus. In: Prechtl HFR, ed. Continuity of neural functions from prenatal to postnatal life. Spastics International Medical Publications, Oxford: Blackwell, 1984: 65-78.

5 Arduini D, Rizzo G, Giorlandino C, Valensise $H$, Dell'Acqua S, Romani C. 1986. The development of fetal behavioural states: a longitudinal study. Prenat Diagn 1982
6:117-24.

Manning FA, Baskett TF, Morrison I, Lange I. Fetal biophysical profile scoring. A prospective study in 1184 high risk patients. Am f Obstet Gynecol 1980;140:289-94.

7 Schmidt W, Boos R, Gnirs J, Auer L, Schulze S. Fetal behavioural states and controlled sound stimulation. Early Hum Dev 1985;12:145-53.

8 van Eyck J, Wladimiroff JW, Noordam JMJ. The blood flow velocity waveform in the fetal descending aorta; its relationvelocity waveform in the fetal descending aorta; its relationship to the fetal behavioural states in normal p.

9 van Eyck J, Wladimiroff JW, Noordam MJ, Tonge HM. The blood flow velocity waveform in the fetal descending aorta; its relationship to behavioural states in the growth retarded fetus at 37-38 weeks gestation. Early Hum Dev 1986;14: 99-107.

10 van Eyck J, Wladimiroff JW, van den Wingaard JAGW, Noordam MJ, Prechtl HFR. The blood flow velocity waveform in the fetal internal carotid and umbilical artery; its relation to fetal behavioural states in normal pregnancy at 37-38 weeks. Br f Obstet Gynaecol 1987;94:736-41.

11 Prechtl HFR. The neurological examination of the full-term newborn infant. Clinics in developmental medicine No 63. 2nd ed. Spastics International Medical Publications. Oxford:
Blackwell, 1977.

12 Brazelton TB. Neonatal behavioural assessment scale. Clinics in developmental medicine No 50. Spastics International

Medical Publications. Oxford: Blackwell, 1973.
13 Dubowitz L, Dubowitz V. The neurological assessment of the pre-term and full-term newborn infant. Clinics in developmenPublications. Oxford: Blackwell, 1987. 